\title{
EchoGéo
}

$57 \mid 2021$

Les enjeux de l'alimentation en eau potable des villes

\section{Formes, facteurs et importance de la vulnérabilité hydrique dans une métropole européenne}

Le cas de Bruxelles

Xavier May, Pauline Bacquaert, Jean-Michel Decroly, Léa de Guiran, Chloé Deligne, Pierre Lannoy et Valentina Marziali

\section{CpenEdition}

\section{Journals}

Édition électronique

URL : https://journals.openedition.org/echogeo/22098

DOI : $10.4000 /$ echogeo.22098

ISSN : 1963-1197

Éditeur

Pôle de recherche pour l'organisation et la diffusion de l'information géographique (CNRS UMR 8586)

Référence électronique

Xavier May, Pauline Bacquaert, Jean-Michel Decroly, Léa de Guiran, Chloé Deligne, Pierre Lannoy et Valentina Marziali, « Formes, facteurs et importance de la vulnérabilité hydrique dans une métropole européenne », EchoGéo [En ligne], 57 | 2021, mis en ligne le 15 octobre 2021, consulté le 08 novembre 2021. URL : http://journals.openedition.org/echogeo/22098 ; DOI : https://doi.org/10.4000/echogeo. 22098

Ce document a été généré automatiquement le 8 novembre 2021.

EchoGéo est mis à disposition selon les termes de la licence Creative Commons Attribution - Pas d'Utilisation Commerciale - Pas de Modification 4.0 International (CC BY-NC-ND) 


\title{
Formes, facteurs et importance de la vulnérabilité hydrique dans une métropole européenne
}

\author{
Le cas de Bruxelles
}

\author{
Xavier May, Pauline Bacquaert, Jean-Michel Decroly, Léa de Guiran, Chloé \\ Deligne, Pierre Lannoy et Valentina Marziali
}

Projet HyPer (L'Hygiène personnelle hors de/sans/mal chez soi), Université Libre de Bruxelles : https://www.ulb.be/fr/region-de-bruxelles-capitale/anticipate-projet-de-recherche-hyper. Ce projet a reçu un financement pour 4 ans de la part d'Innoviris qui est l'institut d'encouragement de la recherche scientifique et de l'innovation de la Région de Bruxelles-Capitale.

1 En 2013, l'Assemblée générale des Nations Unies a adopté à l'unanimité une résolution sur le droit de l'homme à l'eau potable et à l'assainissement. Celle-ci rappelle que «le droit à l'eau potable et à l'assainissement en tant que droit de l'homme découle du droit à un niveau de vie suffisant et est inextricablement lié au droit au meilleur état de santé physique et mentale possible, ainsi qu'au droit à la vie et à la dignité. » Le droit à l'eau est unanimement et universellement consacré comme droit contraignant.

2 Pourtant, au sein même des villes des pays riches, où le droit à l'eau est tenu pour acquis, de nombreuses personnes font face à des difficultés pour accéder à l'eau d'hygiène (González-Gómez, 2020 ; Bony et al., 2021). Par ce terme, nous entendons l'eau pour se laver, l'accès à une toilette ou le lavage du linge. D'une part, un nombre croissant de ménages peinent à payer l'eau du réseau et sont, dans certains cas, privés d'un accès à celui-ci (voir par exemple: Coutard, 1999, pour la France et la GrandeBretagne ; Mack et Wrase, 2017, pour les aires urbaines aux États-Unis ; March et Sauri, 2017 dans le cas de Barcelone; Tsanga Tabi, 2018, pour les villes françaises; Wescoat, 2007 pour Denver). D'autre part, faute d'une offre suffisante d'infrastructures sanitaires offrant un accès public à l'eau d'hygiène, les personnes qui n'ont pas de logement ou qui habitent un logement dont les équipements sanitaires de base font défaut ou ne fonctionnent pas rencontrent de multiples difficultés d'accès à l'eau (voir par exemple : DeMyers et al., 2017, pour les sans-abris à Phoenix, Arizona ; Rosa, 2016, pour les Roms à 
Turin et Marseille ; Speer pour les personnes sans logis vivant dans les camps autour de Fresno, Californie). Ainsi, en 2019, 1,6 \% de la population de l'Union Européenne (UE 27) n'a ni douche, ni baignoire, ni une toilette intérieure avec chasse d'eau (Eurostat, 2021) ; cela concerne plus particulièrement la Roumanie $22,4 \%$, la Lituanie $8,7 \%$, la Lettonie 7,7 \%, la Bulgarie 7,5 \%, l'Estonie 3,5 \% et l'Albanie 3,1\%.

La crise sanitaire liée à l'épidémie de la covid-19 souligne encore davantage la nécessité d'un accès à l'eau pour tou'te-s. S'il était et demeure impératif de demander à la population de se laver régulièrement les mains, encore faut-il que chaque personne puisse disposer des conditions matérielles pour que ce geste, d'apparence si simple, puisse être réalisé.

4 Les recherches citées abordent les difficultés d'accès à l'eau d'hygiène en ville de manière fragmentaire. Certaines se focalisent sur les difficultés de paiement de la facture d'eau par les ménages qui disposent d'un logement, en mobilisant le concept de précarité hydrique calqué sur celui de précarité énergétique (Bradshaw et al., 2013). D'autres, mettent l'accent sur les problèmes rencontrés par certains groupes spécifiques (personnes sans domicile fixe, migrants sans-papier, Roms), qui ne disposent pas de logement ou dont le logement n'est pas équipé d'infrastructures sanitaires. Il en résulte une vision fragmentaire, partielle, de l'inaccessibilité à l'eau. Le présent article vise à surmonter cette difficulté. Dans cette optique, nous proposons tout d'abord de substituer le concept de vulnérabilité hydrique à celui de précarité hydrique. Par ce biais, nous nous donnons les moyens de tenir compte simultanément des deux formes principales de difficultés d'accès à l'eau d'hygiène : celles liées à son coût et celles liées au manque d'équipements sanitaires offrant un accès pour tou'te's à l'eau. Ensuite, en croisant des sources variées, nous objectivons dans le cas de Bruxelles, ces différentes formes de vulnérabilité hydrique et identifions leurs principaux facteurs. À l'aide d'une série d'indicateurs, nous mettons en évidence que, dans un contexte de forte paupérisation, de plus en plus de ménages ont des difficultés à faire face à leurs dépenses en eau. Nous documentons par ailleurs le manque évident d'infrastructures sanitaires accessibles à tou'te's (douches, fontaines, toilettes, lieux pour laver son linge). Finalement, nous proposons une quantification du nombre de personnes en vulnérabilité hydrique à Bruxelles.

\section{La vulnérabilité hydrique : une notion pour saisir les formes multiples de l'inaccessibilité à l'eau}

5 Lorsqu'il est question des difficultés d'accès à l'eau courante pour les ménages, la notion de précarité hydrique s'impose le plus souvent. Dérivée du concept de précarité énergétique, lui-même issu du terme fuel poverty qui a émergé au Royaume-Uni dans les années 1970, la précarité hydrique est communément définie comme une situation où une personne n'a pas accès à de l'eau en quantité suffisante ou de qualité adéquate pour subvenir à ses besoins de base: alimentation, hygiène corporelle, nettoyage des vêtements et entretien du logement. L'opérationnalisation de la définition va toutefois conduire à en restreindre fortement la portée, puisque le terme va être réservé à la description des difficultés d'accès à l'eau dans le logement sous le prisme du poids de la dépense en eau dans le budget du ménage. Sur cette base, les travaux empiriques considèrent généralement, dans le contexte des pays riches, que les ménages sont en précarité hydrique lorsque la dépense en eau dépasse un seuil variant entre $2 \%$ et $5 \%$ 
du revenu (diminué éventuellement du coût du logement) (voir par exemple : Coene et al., 2020; Van Vooren, 2019; Bradshaw et al., 2013; Sociaal-Economische Raad van Vlaanderen, 2012). Selon nous, ce mode d'opérationnalisation de la définition de la précarité hydrique pose problème à plusieurs titres.

Premièrement, il conduit à n'aborder la précarité hydrique que sous l'angle des difficultés de paiement de la dépense en eau. Or, les personnes qui n'ont pas de douche ou de baignoire dans leur logement, pas de toilette, pas d'eau chaude courante voire pas de logement du tout ont un accès (très) problématique à l'eau d'hygiène mais pas nécessairement de difficulté de paiement de leur dépense en eau.

Deuxièmement, un indicateur comme la dépense en eau divisée par le revenu du ménage est trop simple. Il tient éventuellement compte du coût du logement, mais non du patrimoine des ménages. En outre, quel que soit le seuil défini, l'indicateur sélectionne de fait les ménages qui ont une dépense en eau élevée et laisse penser que ce sont ceux-là qui éprouvent des difficultés à payer leur dépense en eau, ce qui n'est pas nécessairement vrai. Certains ménages avec de faibles revenus et un patrimoine important (souvent des personnes âgées) peuvent être considérées comme en précarité hydrique alors qu'ils n'ont aucune difficulté à payer leur dépense en eau.

8 Troisièmement, les ménages qui rationnent exagérément leur consommation d'eau pour rester en mesure de payer leur dépense ne seront pas considérés comme étant en précarité hydrique (puisque leur facture d'eau sera faible), alors que leur dépense en eau est un problème pour eux.

Pour toutes ces raisons, la notion de précarité hydrique telle qu'elle a été utilisée jusqu'à présent nous semble partielle et inadéquate. Nous préférons utiliser le terme de vulnérabilité hydrique que nous définissons comme une situation dans laquelle se trouve une personne qui a des difficultés d'accès à l'eau - pour se laver, laver son linge ou accéder à des toilettes - que ce soit pour des raisons liées au coût de l'eau, aux caractéristiques du logement (absence de toilette, de douche/baignoire, absence d'eau chaude courante, problème de décompte, fuite...) ou à l'absence de logement. En ce sens, la vulnérabilité hydrique est aussi liée à la carence d'infrastructures sanitaires (douches, toilettes, fontaines, laveries) accessibles à tous. Le terme utilisé permet de souligner la diversité des circonstances qui conduisent à la privation d'un droit essentiel. Il convient toutefois de l'utiliser avec prudence, en lui restituant une dimension politique que le paradigme de la capabilité (empowerement) a tendu à effacer ces dernières décennies (Genard, 2014). En effet, nous ne souscrivons pas à l'idée que les personnes faisant face à la vulnérabilité hydrique sont responsables de cette situation et des conséquences qui en résultent pour elles et qu'elles disposeraient des ressources pour s'en sortir. À nos yeux, la notion de vulnérabilité hydrique n'est pas un cache-sexe servant à masquer les rapports de domination et les processus de relégation qui la produisent.

10 En changeant de terminologie, nous voulons souligner la nécessité de prendre en compte tous les publics qui ont des problèmes d'accès à l'eau, sans se focaliser exclusivement sur les difficultés de paiement ou les coupures d'eau qui ne constituent qu'un aspect du phénomène. Ce sont les réalités observées dans le contexte bruxellois qui appellent à cette indispensable conversion du regard. 


\section{Le contexte bruxellois}

'én de Bruxelles-Capitale s'étend sur 19 communes (municipalités) parm lesquelles la 'Ville de Bruxelles' est la plus vaste et la plus peuplée. Cette dernière englobe le nord-est de la Région, le centre historique (appelé le «Pentagone»), une portion à l'est de ce centre et une extension vers le sud (illustration 4). Dans cet article, lorsque nous parlons de Bruxelles, nous désignons la Région de Bruxelles-Capitale (et non 'la Ville de Bruxelles', qui sera explicitement mentionnée).

point de vue institutionnel, la Région de Bruxelles-Capitale est en charge de la politique de l'eau mais ce sont les communes qui fournissent les équipements sanitaires publics tels que fontaines, toilettes et douches. Nous verrons toutefois qu'il existe des différences importantes entre elles en termes de services offerts.

a distribution de l'eau est assurée par la société Vivaqua, une intercommunale publique sous statut de société coopérative à responsabilité limitée (SCRL). Elle est en charge de la production, de la distribution, de la gestion du réseau d'égouts et de la lutte contre les inondations dans la capitale. Vivaqua peut couper la fourniture d'eau d'un ménage après autorisation du juge de paix. Par contre, les dispositifs visant à limiter le débit de l'eau ne sont pas autorisés à Bruxelles. Dans les faits, la coupure d'eau est utilisée par le distributeur comme un moyen de pression pour obtenir des clients le paiement de leurs dettes puisque l'eau n'est rétablie que lorsque l'entièreté de la dette est remboursée. Ceci peut conduire à une situation où un ménage reste longtemps sans eau, tant qu'il n'a pu rembourser le montant exigé.

14 À Bruxelles, un tiers des ménages est approvisionné par un compteur individuel et deux tiers par un compteur collectif, c'est-à-dire un compteur partagé par plusieurs ménages. Seuls les compteurs individuels peuvent être coupés (il est interdit de couper un compteur d'eau collectif).

\section{Une ville en voie de paupérisation}

15 Au cours des vingt dernières années, la précarité a augmenté sensiblement à Bruxelles. Les revenus des Bruxelloise's ont tendance à croitre beaucoup moins vite que la moyenne nationale. En effet, même si les statistiques fiscales belges ne sont pas exemptes de certains biais ${ }^{1}$, la baisse relative du revenu total net imposable bruxellois par rapport à la moyenne nationale reflète un appauvrissement relatif de la population. En 2000, le revenu total net imposable moyen par habitant était de $10 \%$ inférieur à la moyenne nationale. En 2018, la position relative des Bruxellois s'était encore détériorée avec un revenu total net imposable moyen par habitant inférieur de $22 \%$ à la moyenne nationale (illustration 1 ).

EchoGéo, 57 | 2021 
Illustration 1 - Évolution du revenu total net imposable bruxellois par rapport à la moyenne nationale $(2000-2018$, Belgique $=100)$

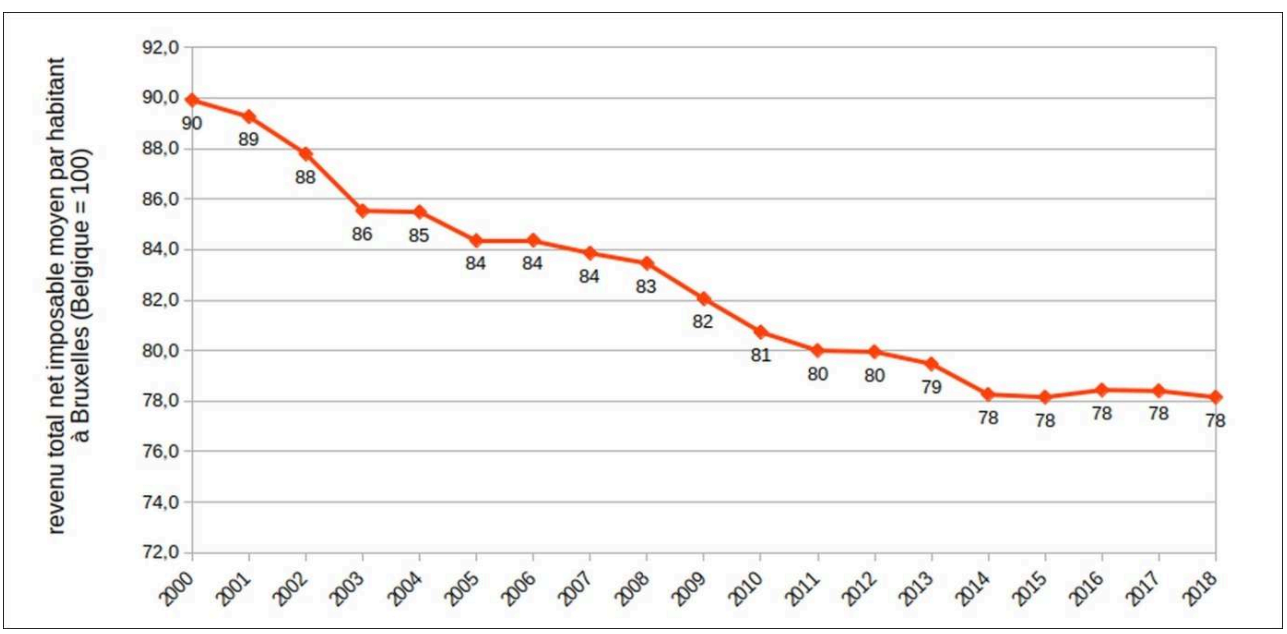

Source : Institut Bruxellois de Statistique et d'Analyse.

Dans le même temps, le coût du logement a fortement augmenté. Selon les données de l'observatoire des loyers ${ }^{2}(2019$, p. 15), le loyer médian a augmenté de $24 \%$ en termes réels à Bruxelles entre 2004 et 2018. De même, le prix de vente médian d'un appartement a doublé en termes réels entre 2000 et $2018^{3}$, soit une croissance beaucoup plus forte que dans les deux autres régions du pays, la Flandre et la Wallonie, d'autant que les prix initiaux étaient déjà plus élevés à Bruxelles.

Par conséquent, on observe simultanément une baisse relative des revenus et une hausse importante du coût du logement. La conjonction de ces deux phénomènes explique notamment l'augmentation de la pauvreté à Bruxelles au cours des vingt dernières années.

Deux autres chiffres permettent de cerner l'ampleur de la pauvreté dans la capitale : un tiers des Bruxellois·es vivent avec un revenu inférieur au seuil de risque de pauvreté (Observatoire de la Santé et du Social de Bruxelles-Capitale, 2019, p.17) et un quart doivent vivre avec en moyenne $8 €$ par jour par personne après avoir payé leurs frais de logement (en ce compris l'eau, le gaz, le chauffage et l'électricité) pour faire face à l'ensemble de leurs dépenses ${ }^{4}$.

19 La pauvreté est donc de plus en plus prégnante dans la Région de Bruxelles-Capitale. Cette évolution a des répercussions importantes sur la capacité des ménages à payer leur dépense en eau dans un contexte d'augmentation du prix de l'eau et de réduction des possibilités d'accès à un logement décent muni des équipements sanitaires de qualité élémentaire. En outre, on observe une augmentation du nombre de personnes qui n'ont pas de logement, nous y reviendrons dans la suite de l'article.

\section{Difficultés d'accès à l'eau en lien avec son coût}

Parmi les ménages qui ont un logement et des équipements sanitaires - eau chaude courante, douche/baignoire et toilettes - en état de fonctionnement, certains peuvent rencontrer des difficultés d'accès à l'eau en raison du poids que représente leur dépense en eau pour leur budget. Par dépense en eau, nous entendons le montant que doivent payer les ménages pour l'eau dans leur logement. Cette dépense est payée soit 
directement à l'intercommunale distributrice lorsque le logement est équipé d'un compteur individuel, soit au gestionnaire du compteur si le ménage est fourni par un compteur collectif. Dans ce dernier cas, il n'y a donc pas de facture d'eau à proprement parler.

21 À Bruxelles, depuis 2005 , la tarification de l'eau est progressive : elle comprend quatre tranches de prix en fonction des volumes d'eau consommés et tient compte du nombre de personnes domiciliées dans le logement au $1^{\mathrm{er}}$ janvier de l'année considérée. En d'autres termes, les seuils entre les différentes tranches de prix sont exprimés en $\mathrm{m}^{3}$ par personne par an. À cela, il faut ajouter une redevance d'abonnement par logement qui est de 25,23€ depuis de nombreuses années.

En raison de cette structure de prix progressive et de la redevance d'abonnement, il n'est pas simple de caractériser l'évolution du prix de l'eau à Bruxelles. Pour ce faire, on utilise souvent l'exemple d'un ménage moyen de deux personnes (la taille moyenne d'un ménage est de 2,17 personnes à Bruxelles en 2020) ayant une consommation moyenne de $35 \mathrm{~m}^{3}$ par personne par an.

Illustration 2 - Évolution du prix de l'eau et du nombre de coupures (Bruxelles, 2000-2021)

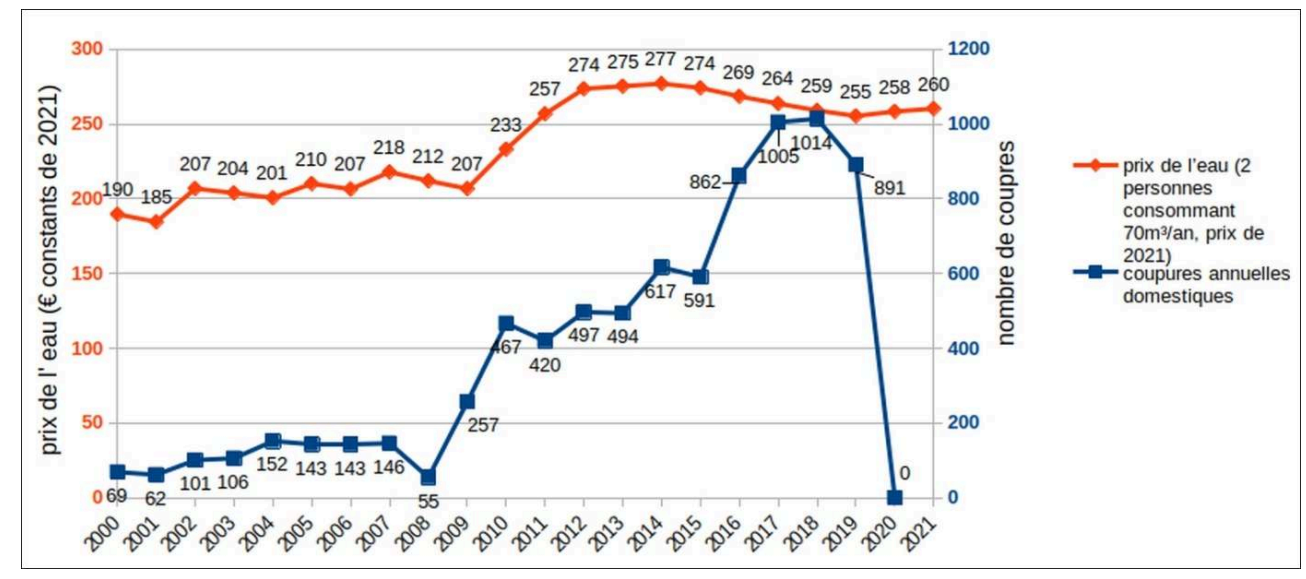

Sources : Vivaqua, Statbel.

Pour un tel ménage moyen, la dépense en eau a augmenté en termes réels de $37 \%$ entre 2000 et 2021 (illustration 2). Il s'agit d'une hausse sensible pour les ménages disposant de faibles revenus, surtout pour un bien de première nécessité indispensable à la dignité humaine. Jusqu'en juillet 2018, date à laquelle il est devenu possible de s'inscrire en ligne pour obtenir une facture mensuelle par voie électronique, les factures étaient la plupart du temps semestrielles ou annuelles (à partir de 2021, les factures deviennent trimestrielles). Par conséquent, lorsque le logement est équipé d'un compteur individuel, le montant de la facture représente un montant important pour le budget de nombreux Bruxellois. Pour les compteurs collectifs, la fréquence de paiement des dépenses en eau tout comme la base du calcul de cette dépense est à l'appréciation du gestionnaire du compteur.

Toutefois, dans les faits, bon nombre de ménages ne correspondent pas à ce modèle de deux personnes ayant une consommation moyenne. En cas de fuite ou de présence de personne's non domiciliée's dans le logement, la dépense peut grimper rapidement car la quatrième tranche de tarification (pour une consommation supérieure à $60 \mathrm{~m}^{3}$ / personne domiciliée/an) est près de quatre fois plus chère que la première. D'après nos 
enquêtes qualitatives, les cas de fuite sont fréquents chez les locataires qui éprouvent des difficultés à payer leur facture d'eau. Les cas où il y a plus de personnes vivant dans le logement que celles qui y sont légalement domiciliées sont également très nombreux, qu'il s'agisse de ménages qui comptent des personnes en séjour irrégulier, d'étudiants domiciliés chez leurs parents (alors qu'ils n'y habitent pas), d'enfants inscrits dans le domicile de l'autre parent en cas de garde partagée, de déménagements en cours d'année civile (Vivaqua tient compte de la situation au $1^{\mathrm{er}}$ janvier), de personnes hébergées temporairement pour de multiples raisons...

La hausse de la facture d'eau combinée aux facteurs qui viennent d'être décrits a conduit, à partir de 2008, à une forte augmentation du nombre de coupures domestiques d'eau à Bruxelles. Leur nombre est en effet passé de 55 en 2008 à 1014 en 2018 pour redescendre à 891 en 2019 (illustration3) et à 0 en $2020^{5}$. La hausse du nombre de coupures n'illustre cependant qu'une petite partie des difficultés d'accès à l'eau, mais ces coupures ont des conséquences particulièrement dévastatrices sur l'isolement social, la santé physique et mentale et la probable spirale d'endettement qui en découle. En effet, la reprise de la fourniture d'eau n'est rétablie qu'après le paiement intégral des dettes à Vivaqua, ce qui peut prendre du temps.

Illustration 3 - Avis d'interruption de la fourniture d'eau et plans de paiement octroyés par Vivaqua (Bruxelles, 2000-2019)

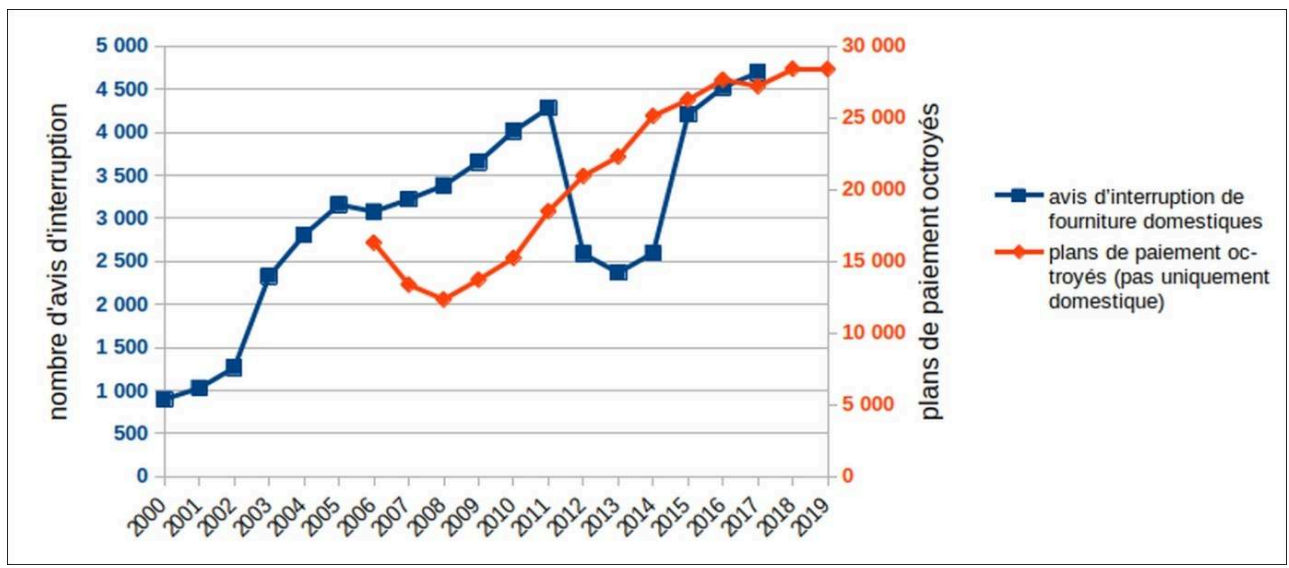

Source : Vivaqua.

Parallèlement, depuis une quinzaine d'années, le nombre d'avis d'interruption de fourniture domestique a aussi fortement augmenté : il est passé de 897 en 2000 à 4694 en $2017^{6}$. Ces avis d'interruption de fourniture correspondent aux ménages qui sont signalés au Centre Public d'Action Sociale (CPAS) de leur commune de résidence car ils n'ont pas payé leur facture d'eau et n'ont pas donné suite à la mise en demeure envoyée 30 jours après l'émission de la facture.

On observe encore que les plans de paiement octroyés par Vivaqua sont en nette progression puisque leur nombre a augmenté de $75 \%$ entre 2006 et 2019. Certes, les abonnés non-domestiques peuvent également bénéficier de plans de paiement mais d'après l'Observatoire de la Santé et du Social de Bruxelles-Capitale (2019, p. 58), ces derniers ne représentent qu'un peu moins de $10 \%$ du total des abonnés, et les cas où ils bénéficient de ces plans de paiement sont rares.

Enfin, le montant des factures échues impayées ne cessent de croitre également. On est ainsi passé de 29 millions $€$ en 2011 à 56,1 millions $€$ en 2019, soit une croissance de $93 \%$ 
en neuf ans. Vivaqua attribue cette hausse en partie à la suppression de la solidarité qui existait jusqu'en 2014 entre propriétaire et locataire. En effet, jusqu'à cette date, l'abonné (généralement le propriétaire) était forcé de prendre en charge les factures d'eau impayées par l'usager (le locataire), y compris pour les compteurs individuels. La règlementation bruxelloise prévoyait donc la solidarité entre " abonné » et " usager » pour le paiement des dettes d'eau, si ceux-ci étaient deux personnes distinctes. Or, en général, les locataires sont moins solvables que les propriétaires. Dès lors, depuis 2014, Vivaqua est plus souvent confronté à des arriérés de paiement.

En résumé, tous les indicateurs précités (prix, coupures, avis d'interruption, plans de paiement, factures échues impayées) vont dans le même sens et attestent qu'un nombre croissant de Bruxellois.e.s rencontrent des difficultés à payer leur facture d'eau depuis au moins une dizaine d'années. Ce constat est largement confirmé par les retours de terrain, comme le souligne par exemple le Centre d'Appui SocialEnergie (2018).

Pourtant, ces problèmes de facture ne reflètent que partiellement les difficultés rencontrées par les ménages. En Région de Bruxelles-Capitale, un tiers des ménages dispose d'un compteur individuel ${ }^{7}$, tandis que les deux autres tiers sont raccordés à un compteur collectif et ne reçoivent donc pas de facture d'eau émanant directement du distributeur. Ces ménages sont absents des statistiques utilisées ci-dessus puisqu'ils n'ont pas de relation avec Vivaqua. Comme il est interdit de couper un compteur collectif à Bruxelles, ils sont protégés des coupures d'eau. Néanmoins, ils peuvent avoir des dettes ou un conflit en lien avec leurs dépenses en eau avec le gestionnaire du compteur collectif (souvent le propriétaire du bâtiment où ils résident). À notre connaissance, il n'existe aucun chiffre sur les dettes de charge des locataires à l'égard de leurs propriétaires à Bruxelles.

Par ailleurs, les problèmes de facture et de difficultés de paiement cachent une autre réalité largement invisible mais régulièrement évoquée par les acteurs de terrain que nous avons rencontrés : la sous-consommation sévère des ménages qui cherchent à minimiser leur dépense en eau pour économiser de l'argent. Ceux-ci n'apparaissent généralement pas dans les statistiques faisant état des difficultés de paiement des factures d'eau.

\section{Difficultés d'accès à l'eau dues au manque d'infrastructures sanitaires}

Outre les difficultés d'accès à l'eau liées au montant des dépenses en eau, il n'est pas toujours possible (ou souhaitable) pour certain'e's d'accéder à l'eau dans un logement. C'est évidemment le cas lorsqu'une personne n'a pas de "chez-soi». Mais d'autres configurations peuvent aussi exister. Une personne peut habiter un logement où il n'y a pas d'eau chaude, de toilette, de douche ou de baignoire en état de fonctionnement. L'eau peut avoir été coupée. On peut préférer ne pas se laver, aller à la toilette ou laver son linge dans le logement qu'on occupe, et ce, pour des raisons extrêmement variées : par souci d'économie, pour ne pas peser financièrement sur l'hébergeur qui offre l'hospitalité, par besoin de contacts sociaux, par peur de se laver seul dans son logement dans le cas de fragilité physique par exemple si on est une personne âgée et isolée... 

Capitale compte seulement trois services publics de douches : la piscine du Centre (2,5 $€)$, la piscine de Laeken $(2,5 €)$, toutes deux sur le territoire de la 'Ville de Bruxelles' et les douches de l'école $n^{\circ} 10$ dans la commune de Molenbeek ( $\left.0,5 €\right)$. Jusqu'en 2017 s'ajoutait à ces trois services celui de la piscine de la commune de Schaerbeek, fermé pour rénovation depuis lors. Enfin, bien que la piscine de la commune de Saint-Josse a rouvert ses portes en 2019 , la remise à neuf de son service de douches est encore à venir. Ces différentes opérations porteront à cinq le nombre de services publics de douches à Bruxelles (soit un service pour 240000 habitants), une situation bien différente de celle de Paris intra-muros avec ses 17 bains-douches gratuits, soit un baindouche pour 130000 habitants (Lévy-Vroelant et Bony, 2019).

Illustration 4 - Carte des douches publiques à Bruxelles (2019)

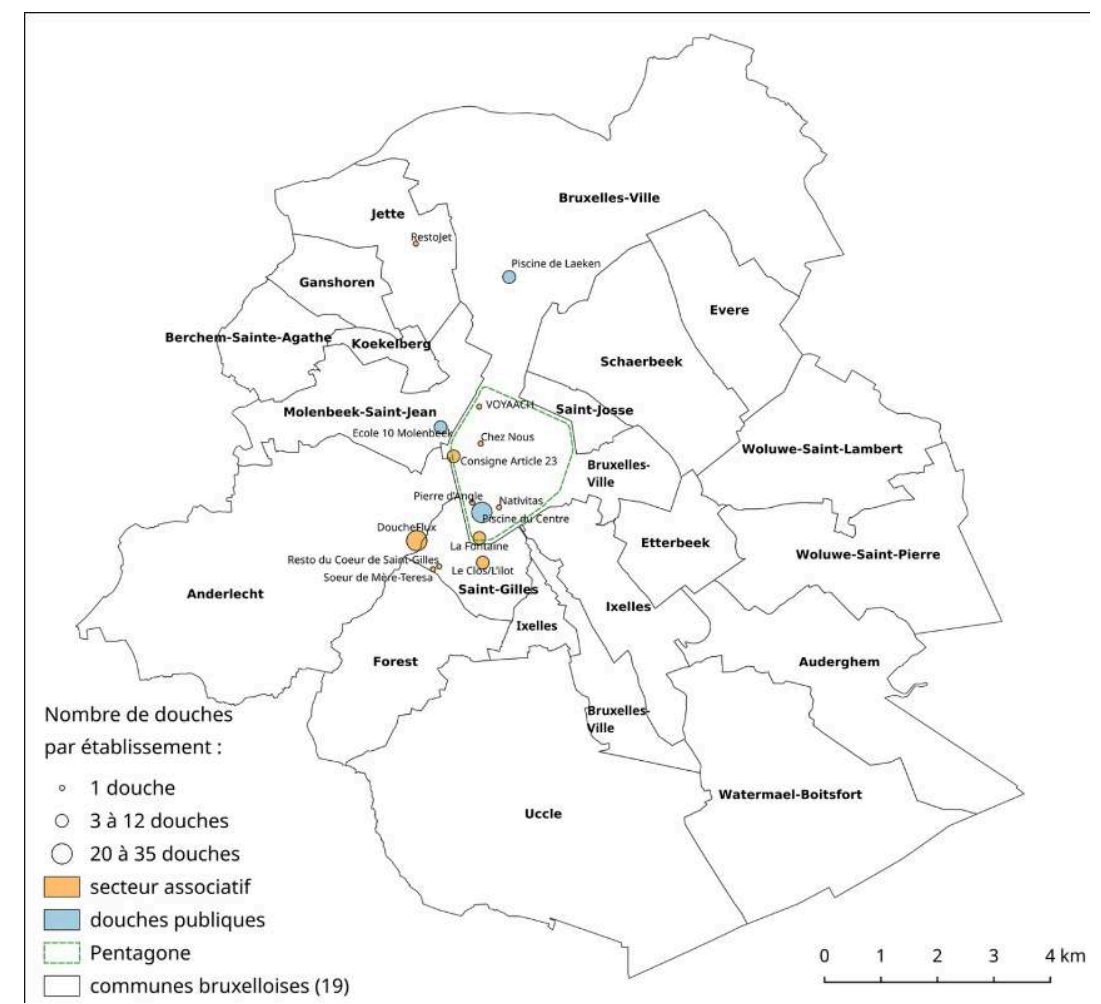

Sources : données collectées par P. Bacquaert et V. Marziali - Carte réalisée en mai 2020

Dans de tels cas de figure, les personnes devraient pouvoir bénéficier d'un accès public à l'eau d'hygiène. Cependant, en la matière, l'offre en Région de Bruxelles-Capitale est dérisoire et très concentrée géographiquement, comme nous allons le voir.

\section{Les douches}

L'offre de lieux publics d'accès à l'eau pour se laver est réduite. La Région de Bruxelles-

Outre les infrastructures publiques, on dénombre à Bruxelles 12 associations offrant la possibilité de se doucher en accueil de jour. Cinq d'entre elles ont pour activité principale la fourniture de douches pour les plus démunie's. Pour chacune d'entre elles, le nombre de cabines s'élève entre 3 et 20, exception faite de Rolling Douche, dont la camionnette mobile ne dispose que d'une seule douche (et n'est dès lors pas 
représentées sur l'illustration 4). Pour les sept autres associations, l'offre de douches reste accessoire (elles disposent d'une seule cabine de douche). douche couvrent des plages de temps limitées. Seules trois des associations recensées donnent la possibilité de prendre une douche l'après-midi. Les autres sont généralement ouvertes entre $9 \mathrm{~h} 30$ et $14 \mathrm{~h} 30$, excluant de facto un grand nombre de personnes qui pourraient $\mathrm{y}$ avoir recours. Ces horaires restreints amplifient l'insuffisance de l'offre, en limitant le nombre potentiel de douches qui peuvent être prises chaque jour.

\section{Les fontaines et les toilettes}

La création récente des associations DoucheFlux (2017), de Rolling Douche (2017) ou l'installation d'une douche dans une association comme RestoJet (dans laquelle la fourniture d'eau n'est pas l'activité principale) témoignent d'une prise de conscience diffuse des besoins croissants en matière d'hygiène corporelle générés par les difficultés d'accès à l'eau. gratuitement ou pour un prix allant de $0,5 €$ à $2,5 €$, ce qui représente 1200 utilisations en moyenne chaque semaine.

proposent des douches, ces lieux sont saturés (c'est moins le cas pour les douches publiques au prix de $2,5 €$ ) dans un contexte où le nombre de sans-abris augmente continuellement. En effet, d'après le dernier dénombrement des personnes sans-abri et mal logées en Région de Bruxelles-Capitale effectué par Bruss'Help, on comptabilisait 2 650 sans-abris en novembre 2020 contre 500 en 2008, leur nombre a donc été multiplié par cinq en douze ans. services de douche sont presque tous situés dans le centre-ouest de la Région, en particulier dans et autour du Pentagone (en pointillé vert sur l'illustration 4), le centre historique de la ville. En revanche, aucun service de douche n'existe dans la plupart des communes bruxelloises. Si on veut avoir accès à une douche publique et qu'on vit loin du centre, il faut à tout le moins faire un long déplacement pour accéder à une infrastructure.

Cette disparité géographique est d'autant plus préoccupante que, selon Quittelier \& Bertrand (2019), on observe un glissement centrifuge de la population des sans-abris dans l'espace public. Les sans-abris s'éloignent de l'environnement immédiat des gares et du centre-ville : $16 \%$ des sans-abris étaient dénombrés hors du Pentagone en 2008 tandis qu'ils étaient $54 \%$ en 2018.

D'autre part, les horaires d'ouverture des associations qui fournissent des services de reste elle aussi très inégale. 
Illustration 5 - Carte des fontaines, toilettes et urinoirs publics à Bruxelles (2019)

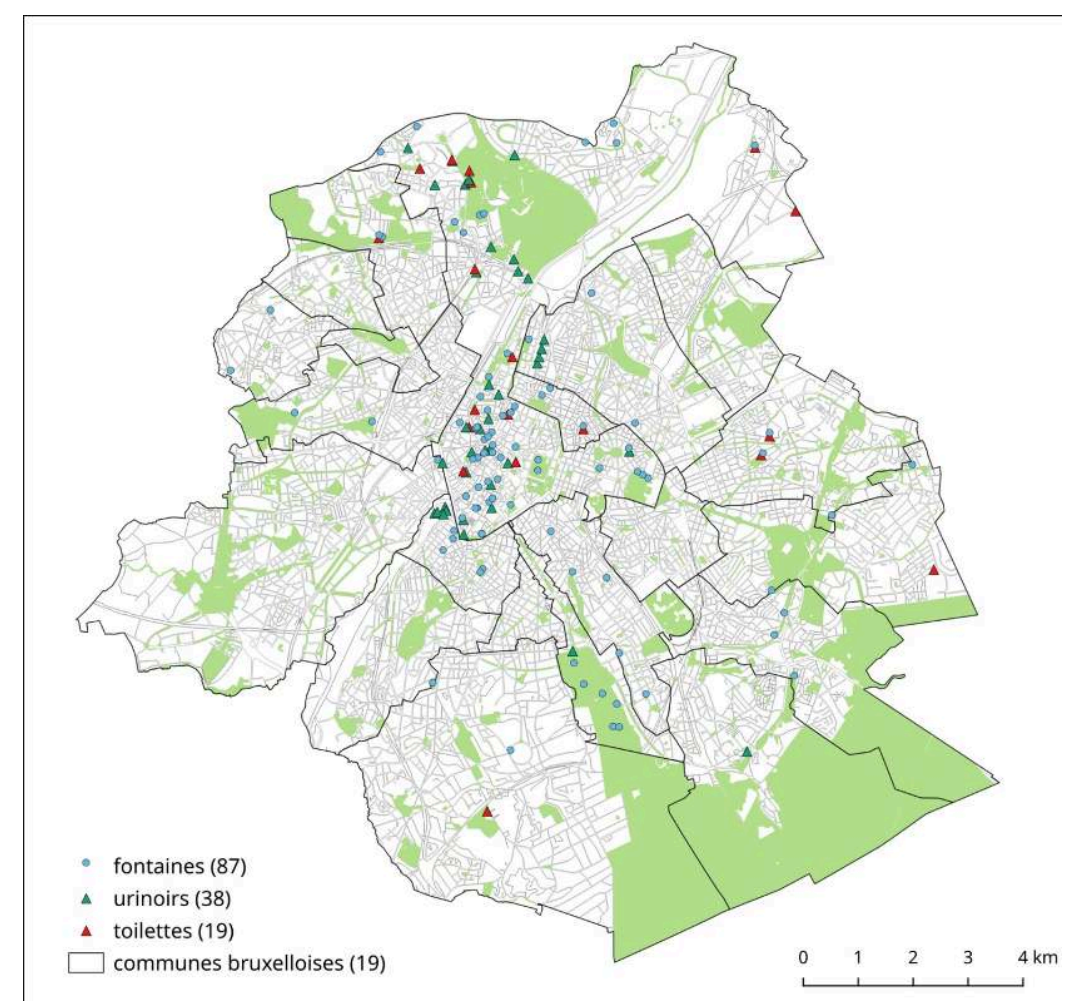

Sources : Infirmiers de Rue, Open Street Map, relevés de terrain effectués par Léa de Guiran.

En effet, la plupart des fontaines, toilettes et urinoirs sont localisés sur le territoire de la 'Ville de Bruxelles' et en particulier dans ou à proximité du Pentagone.

Beaucoup de communes bruxelloises ne proposent pas la moindre toilette publique. Les fontaines publiques sont un peu mieux réparties sur le territoire de la Région de Bruxelles-Capitale mais elles restent fermées six mois par an pour cause d'entretien et de protection contre le gel. Elles sont donc pensées comme un point d'eau pour se déshydrater en été et non comme un service universel et gratuit d'accès à l'eau. La forme des fontaines nouvellement installées par Vivaqua est également parlante : elles sont davantage prévues pour remplir une petite bouteille d'eau qu'un bidon (illustration 6). 
Illustration 6 - Fontaine d'eau Vivaqua

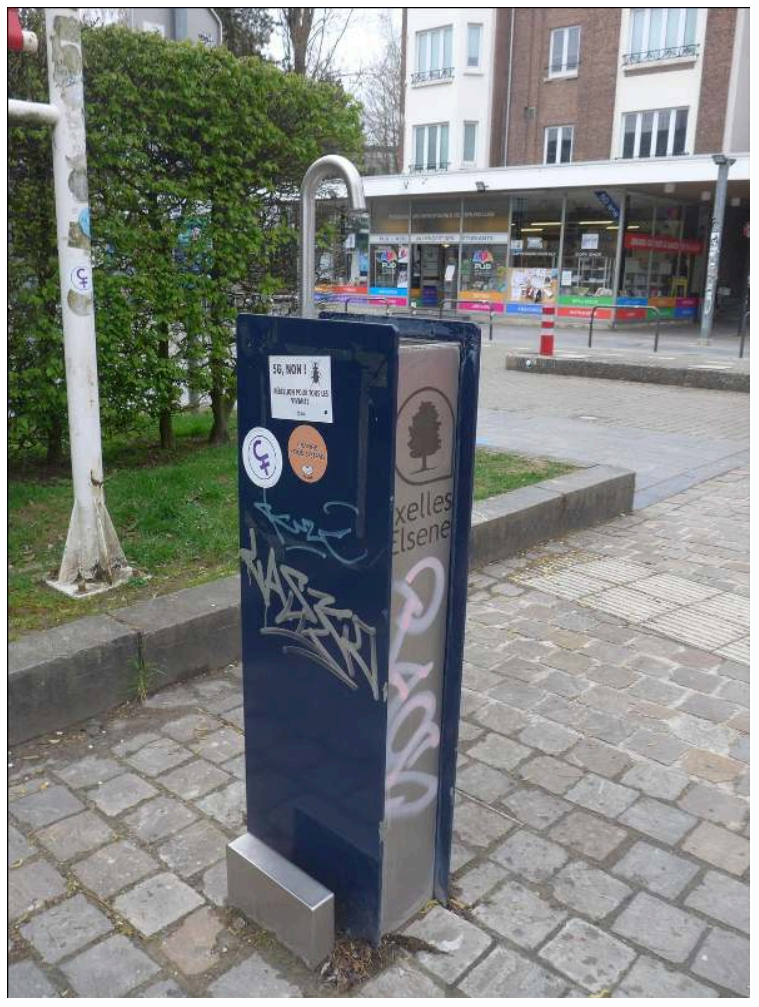

Au croisement avenue Paul Héger et Avenue Buyl à Bruxelles (commune d'Ixelles).

Auteur: Xavier May, avril 2021.

45 L'accès public à l'eau ou à des toilettes est donc très loin d'être garanti à Bruxelles. Ceci est tellement vrai que certains Centres Publics d'Action Sociale (par exemple celui de la commune de Schaerbeek) distribuent des bouteilles d'eau aux habitants qui n'ont pas/ plus l'eau chez eux.

\section{Les laveries automatiques et les associations qui lavent le linge}


Illustration 7 - Carte des laveries automatiques et des associations pour sans-abris qui lavent le linge à Bruxelles (2017)

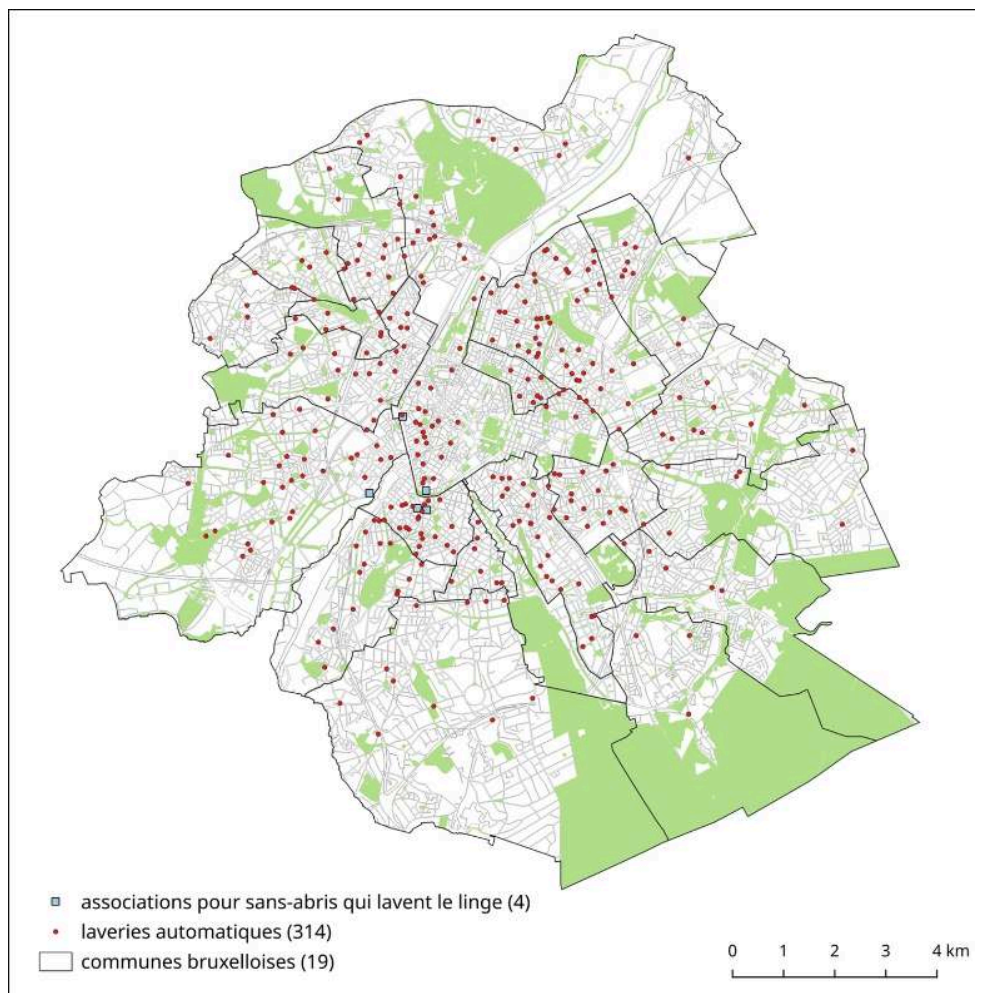

Sources : hub.brussels (2017), données collectées par P. Bacquaert. et V. Marziali (2019) - Carte réalisée en avril 2021.

Les laveries automatiques sont mieux réparties sur le territoire régional, excepté dans la moitié est du Pentagone et à l'est de celui-ci (deux zones de forte densité de bureaux) ainsi que dans certains quartiers périphériques (généralement plutôt aisés). Laver son linge hors de chez soi est donc possible sans devoir parcourir de trop longues distances.

Toutefois, pour les sans-abris, la laverie automatique peut ne pas être pratique : outre la question du prix du lavage et du séchage, il faut disposer d'un stock suffisant de vêtements à laver et faire sécher et/ou pour être vêtu le temps que les machines «tournent». À Bruxelles, nous avons dénombré cinq associations où des personnes sans-abris peuvent faire laver leur linge gratuitement ou pour un prix modique (en plus d'une association mobile qui propose un équipement de faible capacité). Elles sont toutes concentrées à proximité de la partie ouest du Pentagone. Cette concentration géographique pose la question de la faisabilité de laver son linge pour les publics les plus précarisés à Bruxelles. Certaine's recourent aux vêtements distribués gratuitement par les associations plutôt que de laver leur linge.

\section{Quantification du nombre de personnes en vulnérabilité hydrique à Bruxelles}

Pour compléter cette analyse, nous proposons une estimation du nombre de personnes en vulnérabilité hydrique à Bruxelles. 
Tableau 1 - Quantification du nombre de personnes en vulnérabilité hydrique

\begin{tabular}{|c|c|c|c|c|}
\hline $\begin{array}{l}\text { Type de vulnérabilité } \\
\text { hydrique }\end{array}$ & $\begin{array}{l}\text { Situation du } \\
\text { ménage }\end{array}$ & $\begin{array}{c}\text { Catégories de personnes prises en } \\
\text { compte }\end{array}$ & $\begin{array}{c}\text { Estimation du } \\
\text { nombre de } \\
\text { personnes } \\
\text { concernées }\end{array}$ & $\begin{array}{l}\text { Catégories de personnes non } \\
\text { prises en compte }\end{array}$ \\
\hline \multirow{2}{*}{$\begin{array}{l}\text { Vulnérabilité hydrique liée au } \\
\text { cout de l'eau }\end{array}$} & $\begin{array}{l}\text { Compteur d'eau in- } \\
\text { dividuel }\end{array}$ & $\begin{array}{l}\text { Ménages qui demandent un plan de } \\
\text { paiement à la société de distribution } \\
\text { de l'eau (source: Vivaqua) }\end{array}$ & 65000 & $\begin{array}{l}\text { Ménages qui n'ont pas de plan de } \\
\text { paiement auprès de la société de dis- } \\
\text { tribution d'eau mais pour qui la fac- } \\
\text { ture d'eau représente une dépense } \\
\text { importante ; ménages qui se ra- } \\
\text { tionnent exagérément ; ménages } \\
\text { dont l'eau a été coupée }\end{array}$ \\
\hline & $\begin{array}{l}\text { Compteur d'eau col- } \\
\text { lectif }\end{array}$ & & & $\begin{array}{l}\text { Ménages pour qui la facture d'eau } \\
\text { représente une dépense importante } \\
\text { et/ou qui ont des difficultés à payer } \\
\text { les charges pour l'eau au titulaire du } \\
\text { compteur d'eau ; ménages qui se ra- } \\
\text { tionnent exagérement }\end{array}$ \\
\hline \multirow{2}{*}{$\begin{array}{l}\text { Vulnérabilité hydrique liée } \\
\text { aux caractéristiques du loge- } \\
\text { ment }\end{array}$} & $\begin{array}{l}\text { Personnes enregis- } \\
\text { trées au registre na- } \\
\text { tional }\end{array}$ & $\begin{array}{l}\text { Personnes vivant dans un logement } \\
\text { sans eau chaude courante, sans } \\
\text { douche/bain avec un revenu infé- } \\
\text { rieur au revenu médian (sur base de } \\
\text { l'enquête SILC) }\end{array}$ & 27000 & $\begin{array}{l}\text { Personnes occupant un logement } \\
\text { précaire (squats, occupations négo- } \\
\text { ciées...) sans les équipements sani- } \\
\text { taires de base }\end{array}$ \\
\hline & $\begin{array}{l}\text { Personnes non en- } \\
\text { registrées au registre } \\
\text { national }\end{array}$ & $\begin{array}{l}\text { Personnes non reprises au registre } \\
\text { national vivant dans un logement } \\
\text { sans eau chaude courante, sans } \\
\text { douche/bain ou sans toilette (sur } \\
\text { base de Shockaert } \text { et al. } 2012 ; \text { Ro- } \\
\text { berfroid et al., 2015) }\end{array}$ & 5500 & $\begin{array}{l}\text { Personnes occupant un logement } \\
\text { précaire (squats, occupations négo- } \\
\text { ciées...) sans les équipements sani- } \\
\text { taires de base }\end{array}$ \\
\hline $\begin{array}{l}\text { Vulnérabilité hydrique liée à } \\
\text { l'absence de logement }\end{array}$ & & $\begin{array}{l}\text { Nombre de personnes sans-abri } \\
\text { (source : Bruss'Help) }\end{array}$ & 2650 & $\begin{array}{l}\text { Personnes hébergées par des amis ou } \\
\text { de la famille et qui préfërent ne pas } \\
\text { utiliser les installations sanitaires de } \\
\text { l'hébergeur pour ne pas peser sur } \\
\text { son budget }\end{array}$ \\
\hline TOTAL & & & 100150 & \\
\hline
\end{tabular}

Quatre catégories de personnes ont été comptabilisées: les ménages munis d'un compteur individuel qui ont obtenu un plan de paiement de la part de la société distributrice, les personnes en séjour régulier dont l'infrastructure sanitaire dans le logement est déficiente (ces ménages sont inclus dans les enquêtes SILC), les personnes en séjour irrégulier dont l'infrastructure sanitaire dans le logement est déficiente (qui sont exclues des enquêtes et qui occupent plus souvent un logement mal équipé) et enfin les sans-abris.

50 - En 2020, plus de 29500 plans de paiement ont été accordés par Vivaqua (contre 12000 en 2008). Ces chiffres ne concernent que les compteurs individuels (pour rappel, seul un tiers des ménages sont équipés d'un compteur d'eau individuel à Bruxelles) puisque les ménages qui sont raccordés à un compteur collectif n'ont pas de relation directe avec le distributeur. Par conséquent, au moins 29500 ménages ayant un compteur individuel ont (eu) des difficultés à payer leur facture d'eau. La taille moyenne d'un ménage bruxellois étant de 2,2 personnes, on peut en déduire qu'au minimum 65000 personnes sont concernées par des problèmes de paiement de factures d'eau. Si on se réfère aux données contenues dans l'enquête SILC (2017), 5,2 \% des ménages bruxellois déclarent ne pas avoir été en mesure de payer leurs factures d'électricité, d'eau, de gaz ou de chauffage pour des raisons financières au cours des douze derniers mois. Cela représente près de 29000 ménages regroupant 87000 personnes. Les ordres de grandeur sont donc comparables mais l'enquête SILC révèle que les ménages qui ont des difficultés à payer leurs factures d'eau et d'énergie sont en moyenne des ménages de trois personnes (donc sensiblement plus grands que la moyenne bruxelloise). L'estimation de 65000 personnes vivant dans un ménage ayant des difficultés à payer leur facture d'eau est donc une estimation a minima.

51 - Sur base de l'enquête SILC (2017), 2,3 \% des Bruxellois vivent dans un logement qui n'a pas l'eau chaude courante et/ou pas de douche/bain et ont un revenu équivalent inférieur à la médiane ${ }^{8}$. Cela représente environ 27000 personnes. Ces ménages n'ont pas nécessairement un problème de paiement de leur dépense en eau mais il est évident qu'ils ont un accès difficile à l'eau d'hygiène dans la mesure où les infrastructures 
sanitaires dans l'espace public sont insuffisantes et mal réparties sur le territoire de la Région de Bruxelles-Capitale.

Nous excluons les ménages qui ont un revenu équivalent supérieur à la médiane car nous supposons que ceux-ci ne disposent pas de l'eau chaude courante ou d'une douche/baignoire pour des raisons autres que la précarité (logement en travaux, panne...).

53 Si on ajoute le critère de disposer d'une toilette intérieure avec chasse d'eau, le pourcentage de Bruxellois ayant un revenu équivalent inférieur à la médiane qui occupent un logement mal équipé (absence d'eau chaude courante et/ou d'une douche/ baignoire et/ou d'une toilette intérieure avec chasse) grimpe à $3 \%$ (soit environ 35000 personnes).

54 - Roberfroid et al. (2015) font l'hypothèse qu'en Belgique, le nombre de personnes en séjour irrégulier varie entre $7 \%$ et $13 \%$ du nombre de résidents étrangers en situation régulière. Si on applique ces chiffres à Bruxelles, il y aurait entre 30000 et 56000 étrangers en séjour irrégulier au 1er janvier 2020 dans la capitale. Selon Schockaert et al. (2012) qui ont réalisé une enquête sur les personnes sans titre de séjour en Belgique, $18,3 \%$ d'entre eux occupent un logement qui ne jouit pas du confort élémentaire : absence d'un bain ou d'une douche, d'une toilette avec une chasse ou de l'eau chaude courante. Par conséquent, si on prend en considération l'estimation basse de 3000 étrangers en séjour irrégulier à Bruxelles, on conclut à une hypothèse extrêmement prudente de 5500 personnes en séjour irrégulier ayant un accès problématique à l'eau (pas de bain/douche ou pas de toilette avec une chasse ou pas d'eau chaude courante) et qui ne sont pas incluses dans les données de l'enquête SILC.

55 - Le dénombrement du 9 novembre 2020 effectué par Bruss'Help recense 28650 sansabris à Bruxelles au sens de la typologie ETHOS $^{9}$ qui sont assurément en vulnérabilité hydrique. Outre ceux-ci, d'autres situations de vie reprises dans le dénombrement (squats, occupations négociées...) sont très probablement associées à une grande vulnérabilité hydrique.

56 Par conséquent, un décompte extrêmement prudent, privilégiant systématiquement les estimations les plus basses, conduit à évaluer à 100000 le nombre de personnes en vulnérabilité hydrique à Bruxelles, ce qui représente $8,2 \%$ de la population totale. Dans cette estimation, certains ménages peuvent avoir été comptés deux fois. Par exemple, un ménage occupant un logement sans eau chaude courante/douche/bain peut avoir un compteur individuel et avoir demandé un plan de paiement à Vivaqua. De même, une personne en séjour irrégulier peut avoir été dénombrée comme sans-abri.

En revanche, notre estimation du nombre de personnes en vulnérabilité hydrique ignore les ménages qui paient leurs factures d'eau avec de grandes difficultés sans obtenir un plan de paiement de la part de Vivaqua, ceux qui sont raccordés à un compteur collectif ( $2 / 3$ des ménages bruxellois) et qui ont des difficultés de paiement vis-à-vis du gestionnaire du compteur, ceux qui parviennent à payer leur dépense en eau moyennant un rationnement exagéré de leur consommation, les sans logement ${ }^{10}$, ceux qui occupent un logement précaire (squats, occupations négociées...) et qui n'ont pas été comptabilisés ici ou ceux qui subissent une coupure d'eau. Les coupures peuvent avoir été précédées d'un plan de paiement: nous évitons ainsi de compter potentiellement deux fois les mêmes ménages. 

Belgique semble occuper une place privilégiée avec une dépense en eau qui accapare une faible part des dépenses totales pour un ménage moyen.

\section{Illustration 8 - Part des dépenses moyennes en eau dans le total des dépenses moyenne par} ménage (2015)

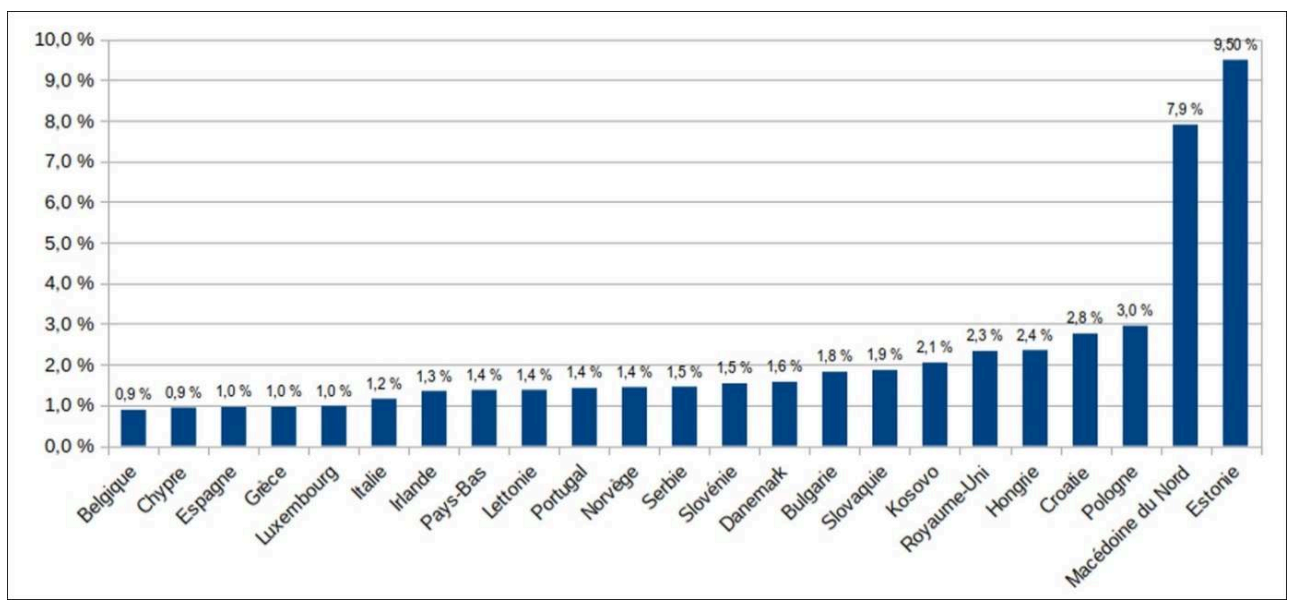

Source : calculs propres sur base des enquêtes sur le budget des ménages (Eurostat).

Sur base de l'illustration 8 , on peut supposer que la précarité hydrique risque d'être plus élevée dans beaucoup plus de pays européens qu'en Belgique.

\section{Conclusions}

Depuis une dizaine d'années, la notion de précarité hydrique a considérablement gagné en visibilité en Belgique. Une recherche exploratoire du Centre d'appui SocialEnergie (2018) a mis le phénomène en lumière. Le milieu associatif et les services sociaux y sont 
de plus en plus souvent confrontés et y sont plus attentifs qu'auparavant comme nous l'ont rapporté plusieurs acteurs de terrain. Cependant, en pratique, la précarité hydrique est réduite à désigner les problèmes de paiement de la consommation d'eau, ignorant la diversité des situations de non accès à l'eau.

Le Parlement de la Région de Bruxelles-Capitale s'est emparé de la question de la précarité hydrique en janvier 2019 et une résolution parlementaire a été rédigée en avril de la même année appelant à poursuivre les travaux sur ce thème et à rassembler un groupe de travail sur le sujet. Toutefois, cette résolution ne traite que du prix de l'eau, de la périodicité des factures, des difficultés de paiement et des coupures d'eau. Cette résolution est symptomatique d'une analyse partielle de la problématique de l'accès à l'eau: elle laisse de côté toutes les personnes qui, pour une raison ou une autre, n'ont pas ou difficilement accès à l'eau d'hygiène à l'intérieur de leur logement et celles qui n'ont pas de logement. La question de l'accès public à l'eau n'y est aucunement abordée.

C'est pourquoi nous mettons en évidence la notion de vulnérabilité hydrique qui envisage l'accès à l'eau pour tous y compris ceux qui n'ont pas de dépense en eau. Dans cette perspective, plutôt que d'utiliser l'indicateur de précarité hydrique qui nous semble inadéquat, nous privilégions une quantification (extrêmement prudente) du nombre de personnes en vulnérabilité hydrique. Le défaut majeur de cette tentative d'estimation est que nous passons à côté des problèmes rencontrés par les ménages raccordés à un compteur collectif. À l'heure actuelle, nous ne voyons malheureusement pas comment remédier à ce défaut. Ceci est d'autant plus regrettable que la question des compteurs collectifs constitue un impensé des politiques publiques d'accès à l'eau alors même que ces compteurs approvisionnent deux tiers des ménages bruxellois. Si les problèmes de fourniture (fuites, tarif, factures...) sont souvent examinés sous l'angle des compteurs individuels, c'est sans doute parce qu'il est plus facile d'imaginer des solutions pour ces cas-là. Nous avons aussi pu constater ce biais dans le groupe de travail (auquel nous participons) mis en place à la suite de la résolution parlementaire de 2019 évoquée ci-dessus: les solutions envisagées ne s'adressent presque exclusivement qu'aux ménages raccordés à un compteur individuel.

Avec au minimum 100000 personnes concernées, soit $8,2 \%$ de la population, la vulnérabilité hydrique constitue dès à présent un problème social important à Bruxelles. De surcroit, les perspectives en la matière sont plutôt sombres. La crise de la covid-19 risque de précariser encore davantage une proportion importante de la population bruxelloise sans compter que l'organe responsable de la régulation du prix de l'eau (Brugel) prévoit une augmentation de ce prix de l'ordre de $20 \%$ en 2022. Bien qu'il soit prévu qu'une intervention sociale visant à réduire cette augmentation soit mise en place pour certaines catégories de ménages, les modalités ne sont pas encore arrêtées au moment d'écrire ces lignes. Toutefois, il est certain que tous les ménages pauvres ne pourront en bénéficier (notamment en raison du problème de non-recours au droit). En revanche, deux éléments pourraient avoir un impact positif sur la situation de certain'e-s Bruxellois'e's : d'une part, il est question d'interdire les coupures d'eau à Bruxelles (comme ce fut le cas durant la crise de la covid-19), mesure qui éviterait de faire basculer certains ménages dans une situation de privation d'eau; d'autre part, la trimestrialisation des factures d'eau a été mise en place en 2021, décision qui permet aux ménages de fractionner davantage le paiement de leur consommation que lorsque la facture est annualisée. 


\section{BIBLIOGRAPHIE}

Barbier R., Gremmel J., 2017. L'accès à l'eau pour tous en France ou la trajectoire erratique d'un problème de second rang (1984-2016). Cahiers de géographie du Québec [En ligne], vol. 61, n 174, p. 427-446. DOI: https://doi.org/10.7202/1053660ar

Bony L., Lévy-Vroelant C, Tsanga Tabi M. (dir.), 2021. Précarités en eau - Un état des lieux en Europe. Aubervilliers, INED, $216 \mathrm{p}$.

Bradshaw J., Huby M., 2013. Water poverty in England and Wales. Journal of Poverty and Social Justice [En ligne], vol. 21, n 2, p. 137-148. DOI: https://doi.org/10.1332/175982713X669835

Brugel, 2021. Motivation vis-à-vis de la méthodologie à respecter par VIVAQUA dans le cadre de la fixation des tarifs à appliquer pour les missions liées à l'eau en Région de Bruxelles-Capitale. Décision (BRUGELDECISION-20210330-152ter), 13 p. [En ligne]. URL: https://www.brugel.brussels/publication/ document/decisions/2021/fr/DECISION-152TER-MOTIVATION-VIVAQUA.pdf

Centre d'Appui SocialEnergie, 2018. Conclusions de la recherche « Accès à l'eau, un droit pour tous? Paroles de naufragés » [En ligne]. URL: https://www.socialenergie.be/wp-content/uploads/ Conclusions_recherche_precahydrique.pdf

Coene J. \& Meyer S., 2020. Baromètres de la précarité énergétique et hydrique (2009-2018). Bruxelles, Fondation Roi Baudouin, 58 p. [En ligne]. URL: https://www.kbs-frb.be/fr/ /media/Files/Bib/ Publications/PUB2020_3700_BarometresPrecariteEnergetiqueHydrique_DEF.pdf

Coutard 0.,1999. L'accès des ménages à faible revenu aux services d'eau et d'énergie en France et en Grande-Bretagne. FLUX, n 36-37, p. 7-15.

De Keersmaecker M.-L., 2019. Observatoire des loyers - Enquête 2018. Bruxelles, Observatoire régional de l'Habitat Région de Bruxelles-Capitale, slrb-bghm.brussels [En ligne]. URL: https:// slrb-bghm.brussels/sites/website/files/publications/documents/slrb-obsloyer-enquete2018-frweb.pdf

DeMyers C., Warpinski C., \& Wutich A., 2017. Urban water insecurity: A case study of homelessness in Phoenix, Arizona. Environmental Justice [En ligne], vol. 10, n 3, p. 72-80. DOI: http://doi.org/10.1089/env.2016.0043.

Eurostat, 2021. Sustainable development in the European Union - Monitoring report on progress towards the SDGs in an EU context, 2021 Edition, Brussels [En ligne]. DOI: https://doi.org/10.2785/195273

Genard J. L., 2014. La question de la responsabilité sous l'horizon du référentiel humanitaire. In Brodiez-Dolino A., von Bueltzingsloewen I., Eyraud B., Laval C., Ravon B., (éd.), Vulnérabilités sanitaires et sociales. De l'histoire à la sociologie. Rennes, Presses Universitaires de Rennes, p. 41-58.

González-Gómez F., García-Rubio M. Á., Guardiola, J., 2020. Some reflections on water for residential uses in developed countries. International Journal of Water Resources Development [En ligne], vol. 36, n² 2-3, p. 311-324. DOI: https://doi.org/10.1080/07900627.2019.1707072

Lévy-Vroelant C., Bony L., 2019. Les bains-douches de Paris : une enquête sur les lieux et leurs usages. Paris, Documents de travail de l'INED n²52, 94 p. [En ligne]. URL: https://www.ined.fr/fichier/ s_rubrique/29360/document_travail_2019_252_bains.douches_eau.fr.pdf

Mack E. A., Wrase S., 2017. A burgeoning crisis? A nationwide assessment of the geography of water affordability in the United States. PloS one [En ligne], vol. 12, $n^{\circ} 1$. DOI: https://doi.org/ 10.1371/journal.pone.0169488 
March H., Saurí D., 2017. When sustainable may not mean just : a critical interpretation of urban water consumption decline in Barcelona. Local Environment [En ligne], vol. 22, n 5, p. 523-535. DOI: https://doi.org/10.1080/13549839.2016.1233528

Observatoire de la Santé et du Social de Bruxelles-Capitale, 2019. Baromètre social 2019. Bruxelles, Commission Communautaire Commune, 82 p. [En ligne]. URL: https://www.ccc-ggc.brussels/fr/ observatbru/publications/2019-barometre-social

Quittelier B., Bertrand F., 2019. Dénombrement des personnes sans-abri et mal logées en Région de Bruxelles-Capitale. Bruxelles, La Strada, 72 p. [En ligne]. URL: http://www.brusshelp.org/images/ LAS3220_Denombrement2018_FR_5_BD.pdf

Roberfroid D., Dauvrin M., Keygnaert I., Desomer A., Kerstens B., Camberlin C., Gysen J., Lorant V., Derluyn I., 2015. Quels soins de santé pour les personnes en séjour irrégulier ? - Synthèse. Bruxelles, Centre Fédéral d'Expertise des Soins de Santé (KCE), 32 p. [En ligne]. URL: https://kce.fgov.be/ sites/default/files/atoms/files/KCE__257B_Soins_de_sante_migrants_Synthese.pdf

Rosa E., 2016. Pratiques discrètes de résistance des migrants roms à Turin et à Marseille. Cultures \& Conflits [En ligne], $\mathrm{n}^{\circ}$ 101, p. 19-34. DOI: https://doi.org/10.4000/conflits.19133

Schockaert I., Morissens A., Cincinnato S., Nicaise I., 2012. Armoede tussen de plooien - Aanvullingen en correcties op EU-SILC voor verborgen groepen armen. Leuven, HIVA, KULeuven, 226 p. [En ligne]. URL: https://lirias.kuleuven.be/1897396

Sociaal-Economische Raad van Vlaanderen, 2012. Sociale aspecten waterbeleid. Brussel, 81 p. [En ligne]. URL: https://www.serv.be/sites/default/files/documenten/

SERV_RAP_20121121_sociale\%20aspecten\%20waterbeleid.pdf

Speer J., 2016. The right to infrastructure : A struggle for sanitation in Fresno, California homeless encampments. Urban Geography [En ligne], vol. 37, n 7, p. 1049-1069. DOI: https:// doi.org/10.1080/02723638.2016.1142150

Tsanga Tabi M., 2018. Analyse des difficultés d'accès à l'eau des ménages en impayé et du ressenti de la qualité de prise en charge de ces ménages par les distributeurs d'eau en France, Institut national de recherche en sciences et technologies pour l'environnement et l'agriculture (IRSTEA), 139 p. [En ligne]. URL: https://hal.inrae.fr/hal-02607572/document

Van Vooren D., 2019. De l'eau pour tous ! État des lieux de la précarité hydrique en Belgique - 2019. Bruxelles, Fondation Roi Baudouin, 24 p. [En ligne]. URL: https://www.kbs-frb.be/fr/Activities/ Publications/2019/20190319NT

Wescoat Jr J. L., Headington L., Theobald R.,2007. Water and poverty in the United States. Geoforum [En ligne], vol. 38, n 5, p. 801-814. DOI: https://doi.org/10.1016/j.geoforum.2006.08.007

\section{NOTES}

1. Les statistiques fiscales belges n'incluent par exemple pas certains revenus de transferts comme les allocations familiales, les revenus d'intégration sociale (ou équivalent), les allocations aux personnes handicapées, etc. De même, l'enregistrement des revenus mobiliers et immobiliers est très partiel.

2. En raison d'un échantillonnage insatisfaisant, les données de l'observatoire des loyers ne sont pas exemptes de biais. Il s'agit toutefois de la seule source existante sur les loyers en Région de Bruxelles-Capitale. 
3. Selon Statbel, le prix médian d'un appartement, studio ou flat est passé de $74368 €$ en 2000 à $210000 €$ au premier semestre 2018 en Région de Bruxelles-Capitale. Si on prend en compte l'inflation sur la période, les prix ont augmenté en termes réels de $99 \%$.

4. Chiffre obtenu sur base des micro-données de l'enquête EU-SILC (Survey on Income and Living Conditions, 2017) qui a pour thème les revenus et les conditions de vie des ménages. En Belgique, elle est réalisée chaque année par Statbel, l'office belge de Statistique. Elle ne concerne que les ménages privés qui sont enregistrés au Registre national: les ménages collectifs comme les foyers, maisons de repos, prisons, communautés religieuses... ne sont pas pris en compte. En 2017, 6054 ménages ont répondu à l'enquête, dont 1076 ménages bruxellois. Ces enquêtes ont généralement lieu en face à face.

5. Le nombre de coupures annuelles dépend également de la politique menée par Vivaqua en termes de récupération de créances. Par exemple, depuis les débuts de la crise de la covid-19 (mars 2020), les coupures d'eau ont été suspendues; c'est pourquoi il y a en a eu 0 en 2020.

6. Les fluctuations du nombre d'avis d'interruption varient en fonction des priorités que Vivaqua établit en termes de gestion du remboursement de ses créances. Les réponses de Vivaqua à nos questions n'ont pas été plus précises.

7. Questions et Réponses - Parlement de la Région de Bruxelles-Capitale - Session ordinaire 2017-2018. 15 janvier 2018 ( $n^{\circ} 36$ ), Question parlementaire $n^{\circ} 684$ de Mme Catherine Moureaux du 6 novembre 2017, p. 139-140. http://weblex.brussels/data/crb/bqr/2017-18/00036/ images.pdf\#page=139

8. Le revenu équivalent correspond au revenu total d'un ménage divisé par le nombre de membres du ménage converti en équivalents adultes. Les équivalents adultes sont estimés de la manière suivante : 1 pour le premier adulte, 0,5 pour chaque autre membre âgé de 14 ans et plus et 0,3 par enfant de moins de 14 ans (conformément à l'échelle d'équivalence modifiée de l'OCDE). Il s'agit donc du calcul d'un revenu qui prend en compte la composition du ménage. En effet, bénéficier d'un revenu de $3000 €$ pour une personne seule ou pour un ménage de quatre personnes conduit à des situations de fait très différentes.

9. La typologie de l'exclusion liée au logement appelée ETHOS (European Typology on Homelessness and housing exclusion) a été développée par la Fédération européenne d'Associations Nationales Travaillant avec les Sans-Abri (FEANTSA). Cette typologie énonce quatre formes d'exclusion liée au logement : être sans abri, être sans logement, être en situation de logement précaire ou être en situation de logement inadéquat.

10. Nous entendons par là toutes les personnes qui ne sont pas sans-abri mais qui n'ont pas de logement. Il peut s'agir par exemple de personnes hébergées en foyer d'hébergement ou en centre d'accueil, de personnes hébergées par des amis ou de la famille, de personnes sortant d'institutions (médicale, pénitentiaire, pour enfant)...

11. Ce seuil de $2,26 \%$ correspond au double du ratio médian pour l'ensemble de la population belge entre les factures d'eau d'une part et les revenus disponibles déduction faite du coût du logement d'autre part. Les données utilisées pour ces estimations proviennent de l'Enquête sur les revenus et les conditions de vie (SILC, 2018) qui est une enquête européenne qui porte sur près de 6000 ménages en Belgique. 


\section{RÉSUMÉS}

Cet article traite des difficultés d'accès à l'eau d'hygiène (pour se laver, accéder à une toilette ou laver son linge) rencontrées par les personnes qui vivent à Bruxelles. Dans ce cadre, nous mettons en évidence la notion de vulnérabilité hydrique qui prend en compte les difficultés d'accès à l'eau en raison de son coût, des caractéristiques du logement (fuite, absence des équipements sanitaires de base...) ou de l'absence de logement. Nous montrons qu'en raison de l'appauvrissement de la population bruxelloise et du manque d'infrastructures sanitaires publiques (en particulier les douches, fontaines et toilettes publiques), au minimum 100000 personnes, soit 8,2 \% de la population bruxelloise, est en vulnérabilité hydrique.

This article deals with the difficulties of access to water for hygiene (for washing, accessing a toilet or washing clothes) encountered by people living in Brussels. We highlight the notion of water vulnerability, which takes into account the difficulties of access to water due to its cost, housing characteristics (leakage, absence of basic sanitary equipment, etc.) or absence of housing. We show that at least 100,000 people, i.e. $8.2 \%$ of the Brussels population, are in water vulnerability because of the impoverishment of the Brussels population and lack of public sanitary infrastructures (in particular showers, fountains and public toilets).

\section{INDEX}

Mots-clés : accès à l'eau, vulnérabilité hydrique, douche publique, toilette et fontaine publique, Bruxelles

Thèmes : Sur le Champ

Keywords : access to water, water vulnerability, public shower and toilet, drinking fountain, Brussels

\section{AUTEURS}

\section{XAVIER MAY}

Xavier May, Xavier.May@ulb.be, Institut de Gestion de l'Environnement et d'Aménagement du Territoire (IGEAT), Université Libre de Bruxelles. Il a récemment publié :

- May X., Bacquaert P., Decroly J.-M., et al., 2021. Pourquoi ne pas en finir avec la tarification progressive de l'eau à Bruxelles ? Brussels Studies [En ligne], n 156. URL: http://

journals.openedition.org/brussels/5494

- May X., Ermans T., Hooftman N., 2019. Les voitures de société : diagnostics et enjeux d'un régime fiscal. Brussels Studies [En ligne], Note de synthèse $n^{\circ}$ 133. URL: http:// journals.openedition.org/brussels/2366

- May X., 2014. Mesure multidimensionnelle de la pauvreté des ménages en Belgique. In Knapen H., Braes S., Ermans T., Herremans W., Le Datawarehouse, un mille-pattes! Perspectives $d u$ Datawarehouse Marché du Travail et Protection Sociale. Gent, Academia Press BELSPO, p, 71-91.

\section{PAULINE BACQUAERT}

Pauline Bacquaert, Pauline.Bacquaert@ulb.be, Laboratoire interdisciplinaire en Etudes urbaines (LIEU), Université Libre de Bruxelles. Elle a récemment publié : 
- Bacquaert P., Decroly J.-M., Deligne C., et al. , 2022. Vulnérabilité hydrique et accès à l'eau d'hygiène : compromis de coexistence à Bruxelles. Espaces \& Sociétés, $\mathrm{n}^{\circ}$ 186-187 (à paraitre). - May, X., Bacquaert, P., Decroly, et al., 2021. Pourquoi ne pas en finir avec la tarification progressive de l'eau à Bruxelles? Brussels Studies [En ligne], nº 156. URL : http:// journals.openedition.org/brussels/5494

- Bacquaert P., 2020. L'aridité des communs. Bruxelles en mouvements [En ligne], $\mathrm{n}^{\circ} 304$. URL: https://www.ieb.be/L-aridite-hybrique-visages-de-la-vulnerabilite-hydrique

\section{JEAN-MICHEL DECROLY}

Jean-Michel Decroly, Jean-Michel.Decroly@ulb.be , Institut de Gestion de l'Environnement et d'Aménagement du Territoire (IGEAT), Université Libre de Bruxelles. Il a récemment publié : - Boussauw K., Decroly J.-M., 2021. Territorializing international travel emissions: geography and magnitude of the hidden climate footprint of Brussels. Urban Planning, vol. 6, n 2, p. 285-298.

- Dobruszkes F., Ramos-Pérez D., Decroly J.-M., 2019. Reasons for Flying. In Graham A., Dobruszkes F. (eds), Air Transport: A Tourism Perspective. Oxford, Elsevier, p. 23-39.

- Wayens, B., Decroly, J-M., Strale, M., et al., 2020. Pedestrianization of a multifunctional space: challenges and early observations on the Brussels Pentagon. In Vermeulen S., Mezoued A. M., De Visscher J.-P. (ed.), Towards a metropolitan city centre for Brussels. Brussels, Editions de l'Université de Bruxelles, VUB Press and Brussels Studies Institute, p. 55-79.

\section{LÉA DE GUIRAN}

Léa de Guiran, lea.deguiran@gmail.com, IGEAT, Université Libre de Bruxelles) ; elle a récemment publié :

- May, X., Bacquaert, P., Decroly, J.-M., de Guiran, L., et al., 2021. Pourquoi ne pas en finir avec la tarification progressive de l'eau à Bruxelles? Brussels Studies [En ligne], n 156. URL: http:// journals.openedition.org/brussels/5494

\section{CHLOÉ DELIGNE}

Chloé Deligne, chloe.deligne@ulb.be, FNRS/Université Libre de Bruxelles. Elle a récemment publié :

- May, X., Bacquaert, P., Decroly, J.-M., de Guiran, L., Deligne, C., et al., 2021. Pourquoi ne pas en finir avec la tarification progressive de l'eau à Bruxelles ? Brussels Studies [En ligne], $\mathrm{n}^{\circ} 156$. URL: http://journals.openedition.org/brussels/5494

- Cahn L., Deligne C., Pons-Rotbardt N., et al., 2018. Terres de villes. Enquêtes potagères de Bruxelles aux premières saisons $d u X X X^{e}$ siècle. Paris, Éditions L'Éclat, 320 p.

- Deligne C., 2017. Les eaux de Bruxelles : systèmes et dépendances. In Lestel L., Carré C. (dir.), Les rivières urbaines et leur pollution (Paris, Berlin, Bruxelles, Milan). Paris, Edition Quae, p. 173-188.

\section{PIERRE LANNOY}

Pierre Lannoy, Pierre.Lannoy@ulb.be, METICES, Université Libre de Bruxelles. Il a récemment publié :

- Demoli Y., Lannoy P., 2019. Sociologie de l'automobile. Paris, Ed. La Découverte, 128 p.

- Gerkens J.-P., Lannoy P., 2020. Brève histoire du vélo racontée depuis Bruxelles. Cahiers de l'Observatoire de la mobilité de la Région de Bruxelles-Capitale, n 7, p. 12-29.

- Lannoy P., Ouali N., 2021. Matérialité de l'espace urbain et égalité des sexes : mesures et enjeux de l'odonymie bruxelloise. Géographie et cultures, n 115, p. 157-179. 


\section{VALENTINA MARZIALI}

Valentina Marziali, Valentina.Marziali@ulb.be, METICES, Université Libre de Bruxelles. Elle a notamment publié :

- Bacquaert P., Decroly J.-M., Deligne C., Lannoy P., Marziali V., May X., 2022. Vulnérabilité hydrique et accès à l'eau d'hygiène : compromis de coexistence à Bruxelles. Espaces \& Sociétés, $\mathrm{n}^{\circ}$ 186-187 (à paraitre).

- May X., Bacquaert P., Decroly J.-M., de Guiran L., Deligne C., Lannoy P., Marziali V., 2021.

Pourquoi ne pas en finir avec la tarification progressive de l'eau à Bruxelles? Brussels Studies [En ligne], $\mathrm{n}^{\circ}$ 156. URL : http://journals.openedition.org/brussels/5494

- Lannoy P., Marziali V., 2012. Les visiteurs et leurs clichés. Figures de l'activité photographique au Musée Autoworld de Bruxelles. In Chaumier S.,Krebs A., Roustan M., Visiteurs photographes au musée. Paris, La Documentation française, p. 115-131. 